\title{
Bien-être animal en élevage de poulets de chair
}

\author{
C. ARNOULD, C. LETERRIER
}

INRA, CNRS, Université de Tours, Haras Nationaux, UMR 85 Physiologie de la Reproduction et des Comportements, F-37380 Nouzilly, France

Courriel : arnould@tours.inra.fr

\begin{abstract}
L'élevage des poulets de chair s'est intensifié ces dernières décennies et de nombreux problèmes de bien-être sont apparus. Cependant, la législation européenne concernant les poulets de chair reste réduite. Au niveau du Conseil de l'Europe, la protection des poulets de chair est traitée dans un texte général qui concerne l'espèce Gallus gallus, c'est-à-dire aussi bien la poule pondeuse que le poulet de chair (recommandation adoptée le 28 novembre 1995 par

maladies parasitaires que ceux élevés en claustration. La mise en cause du bien-être liée aux manipulations réalisées avant l'abattage est traitée dans un autre article de ce numéro spécial (Terlouw et al 2007).

\section{1 / Bien-être des poulets de viande} élevés pour la production
\end{abstract} le Comité permanent de la Convention sur la protection des animaux dans les élevages). Au niveau de l'Union européenne, elle relève d'un texte très général et non normatif qui date de 1998 : la directive $98 / 58 / \mathrm{CE}$ concernant la protection des animaux dans les élevages. En 2000, un rapport scientifique sur le bien-être des poulets de chair a été remis par le Comité Bien-être et Santé animale à la Commission européenne (Anonyme 2000). Il souligne les problèmes de bien-être inhérents aux modes d'élevage actuels. Suite à ce rapport, un projet de directive (Commission européenne 2005), publié en mai 2006, est en cours de négociation.

L'objectif de cette synthèse est de présenter les principaux problèmes de bien-être animal rencontrés en élevage de poulet de chair et identifiés dans le rapport cité plus haut. Cette revue étant en partie basée sur celui-ci, seules les références des travaux non cités dans ce rapport sont mentionnées. Ces problèmes concernent presque exclusivement les poulets de type standard élevés en claustration et les reproducteurs à l'origine de ce croisement terminal. Les poulets de types «certifié» et Label sont très peu concernés. Ces types de production, peu développés par rapport au type de production standard, ne sont cependant pas indemnes d'atteintes au bien-être animal, en particulier en ce qui concerne les problèmes de santé. Les poulets ayant accès à des parcours sont, par exemple, davantage sujets aux
Les poulets destinés à la production de viande sont élevés dans de grands bâtiments et en groupes de plusieurs milliers d'individus. En élevage standard, le nombre d'animaux peut atteindre trente mille. Les poulets sont élevés au sol, soit en claustration (poulet standard), soit avec un accès à un parcours extérieur en fin d'élevage. Dans le cas des poulets standards, ils sont élevés en lumière naturelle ou artificielle. L'intérieur des bâtiments ne comprend que des mangeoires et des abreuvoirs et le sol est recouvert de litière. Les poulets sont abattus à des âges différents selon le type (standard, certifié, Label) et la destination (coquelet, carcasse, découpe). La pression de sélection sur la croissance des poulets standards a été très forte : abattage vers 6 semaines d'âge contre au moins 12 semaines d'âge pour les Label. Les manipulations par l'homme se limitent souvent à la pesée de quelques animaux.

\section{1 / Problèmes de bien-être ren- contrés}

La majorité des problèmes de bienêtre concerne la production de type standard. L'un des plus visibles est la très faible activité des poulets (Arnould et Faure 2004, figure 1), qui augmente avec l'âge (McLean et al 2002) et qui s'accompagne d'une restriction du répertoire comportemental (absence de certains comportements). La sélection pour une croissance très rapide et les pratiques d'élevage telles que la restric- tion en espace, l'environnement peu stimulant (équipements réduits aux mangeoires et abreuvoirs, uniformité de la litière, éclairage continu, alimentation ad libitum), ont probablement conduit à ces modifications comportementales. Les réactions de peur peuvent également provoquer des comportements inadéquats tels que des mouvements de panique, entraînant des étouffements lorsque les animaux sont élevés en grands groupes et à de fortes densités.

Les problèmes locomoteurs, révélateurs d'anomalies musculaires et squelettiques des pattes, constituent une cause majeure de mal-être. Ils affectent la capacité des poulets à marcher, et donc leur facilité d'accès aux équipements, et sont parfois source de douleur. Les lésions cutanées telles que les dermatites de contact peuvent être très fréquentes. Elles affectent essentiellement les pattes (figure 2), mais peuvent aussi se situer au niveau du bréchet. Les pathologies cardio-vasculaires d'origine métabolique peuvent conduire à la mort des animaux. Le confort thermique des poulets peut également être compromis, voire entraîner la mort du fait de la conjonction entre leur forte croissance, qui provoque une forte production de chaleur interne, et certaines pratiques d'élevage comme la restriction en espace, qui limite les possibilités de thermorégulation.

Problèmes comportementaux et problèmes de santé ne sont pas indépendants. Une modification de l'activité des poulets (activité générale, locomotrice...) peut être la cause ou la conséquence de problèmes au niveau des pattes et du revêtement cutané (activité faible : ampoules du bréchet, dermatites ; activité forte : griffures). Ces effets peuvent être directs ou indirects. Une faible activité, par exemple, peut favoriser la présence de dermatites de contact via une détérioration plus rapide de la litière. Elle favorise également une répartition hétérogène des poulets 
Figure 1. Activités, en pourcentage de temps consacré à chaque comportement, de poulets élevés à des densités de 2 poulets $/ m^{2}$ et 15 poulets $/ \mathrm{m}^{2}$ (Arnould et al 2004).

2 poulets $/ \mathrm{m}^{2}$

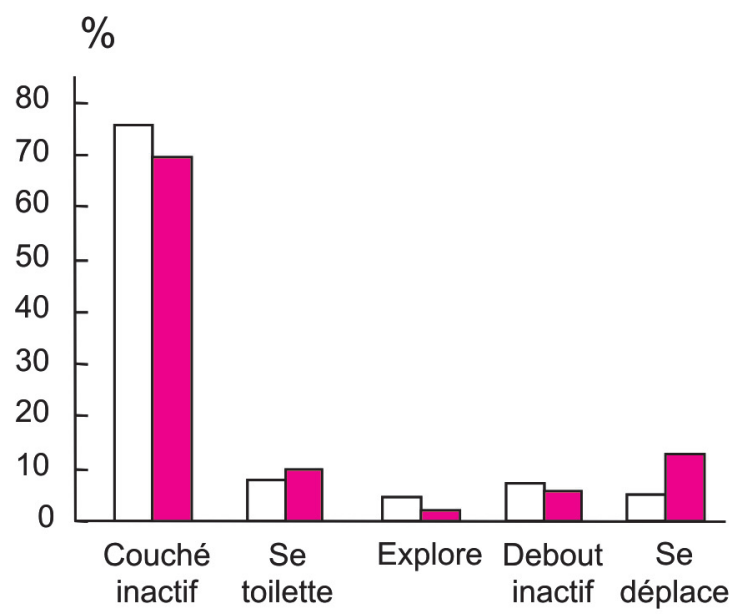

Zone libre
15 poulets $/ \mathrm{m}^{2}$

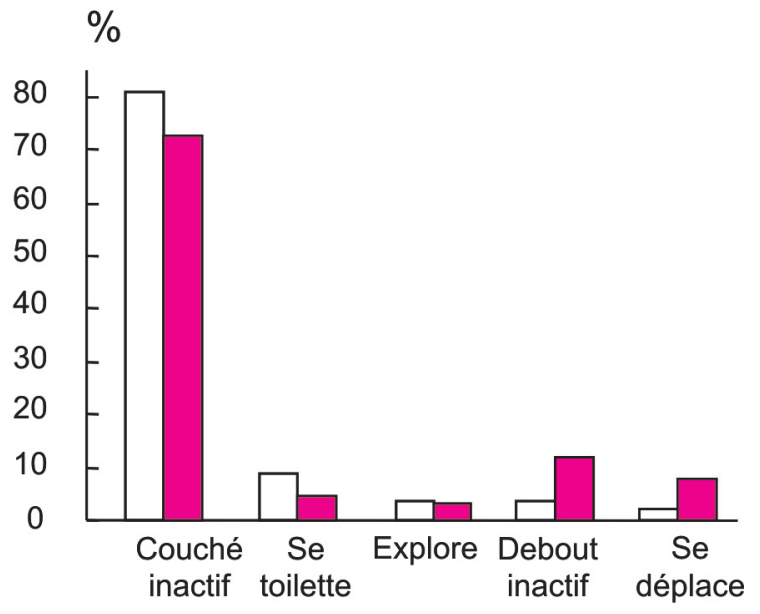

Zone équipements

La surface d'élevage se divisait en une zone vide d'équipement (zone libre) et une zone contenant les mangeoires et les abreuvoirs (zone équipements). Les animaux étaient observés de l'âge de 3 à 6 semaines (âge d'abattage). Les activités liées à l'alimentation et l'abreuvement n'ont pas été prises en compte. Aucun bain de poussière n'a été observé. Entre 3 et 6 semaines d'âge, les poulets passent la grande majorité de leur temps couchés. Ils limitent leurs déplacements, même lorsqu'ils sont élevés à des densités très faibles.

sur la surface d'élevage (Fraysse et al 2001, Arnould et Faure 2003) qui peut ainsi accentuer la dégradation de la litière dans les zones de forte concentration en animaux.

Figure 2. Pododermatite modérée correspondant à la note 6 dans Colas et al 2005.

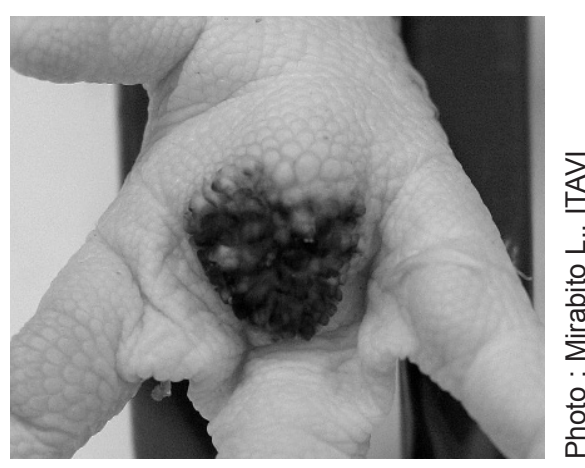

\section{2 / Facteurs influençant le bien-être}

Les études sur l'influence de la densité (nombre d'animaux $/ \mathrm{m}^{2}$ ) ou du chargement (poids $/ \mathrm{m}^{2}$ ) sur le comportement et la santé des poulets sont nombreuses, mais les résultats varient selon les auteurs. Les fortes densités diminuent la vitesse de croissance, mais une ventilation adéquate des bâtiments diminue leur impact. Elles augmentent la mortalité lorsqu'elles sont combinées à d'autres facteurs de stress, comme la chaleur par

exemple. Il en est de même de l'incidence des maladies respiratoires lorsque ces fortes densités sont associées à la présence d'agents infectieux. Elles favorisent les ampoules au bréchet, les dermatites de contact (figure 3 ) et les problèmes locomoteurs. Elles diminuent l'activité générale et locomotrice des poulets et augmentent les dérangements subis par les individus au repos. Il est difficile de définir un seuil de densité/chargement audelà duquel tous ces facteurs sont affectés, car l'impact de la conduite d'élevage sur ce seuil, notamment la capacité à avoir une ventilation adéquate, est considérable (Elwinger 1995, Dawkins et al 2004).

Figure 3. Influence de la densité d'élevage sur le pourcentage de poulets atteints de dermatites au niveau du coussinet plantaire (pododermatites) et des tarses à l'âge de 6 semaines (Martrenchar et al 1997).

$\%$ de poulets atteints de

100 dermatites

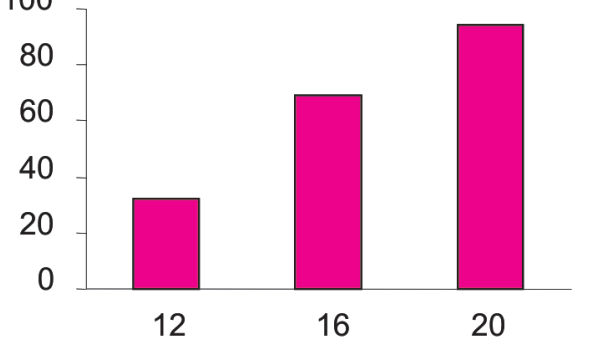

Nombre de poulets $/ \mathrm{m}^{2}$
Les études, peu nombreuses, visant à apporter plus de diversité dans l'environnement des poulets montrent qu'il serait sans doute possible d'améliorer leur bien-être par cette voie. Les effets de l'ajout de perchoirs sont variables, mais en règle générale les poulets se perchent peu. La présence de panneaux verticaux, qui constituent une protection vis-à-vis des congénères ou un lieu où se cacher, conduit à une meilleure répartition des poulets sur la surface d'élevage et diminue la fréquence des dérangements (Cornetto et Estevez 2001, Cornetto et al 2002). L'ajout d'objets, de sable (Arnould et al 2004) ou de paille (Kells et al 2001) stimule l'activité des poulets, mais n'a pas d'effet sur les problèmes locomoteurs. La plupart de ces études ayant été réalisées sur des petits groupes, une évaluation de l'efficacité de ces aménagements et des possibilités d'utilisation en élevage commercial est nécessaire.

La modulation des programmes alimentaires permet de ralentir la vitesse de croissance. Par ailleurs, l'utilisation de séquences de différents aliments permet d'introduire de la diversité dans l'environnement des poulets, de stimuler leur activité et de diminuer l'incidence des problèmes locomoteurs (Bouvarel et al 2004, Leterrier et al 2006 , figure 4). Les effets de cette alimentation dite séquentielle, sont explorés par l'INRA et l'Institut Technique de 
Figure 4. Effet de l'alimentation séquentielle (offrant aux poulets une alternance d'aliments pauvre et riche en lysine) sur le pourcentage de poulets présentant une marche anormale.

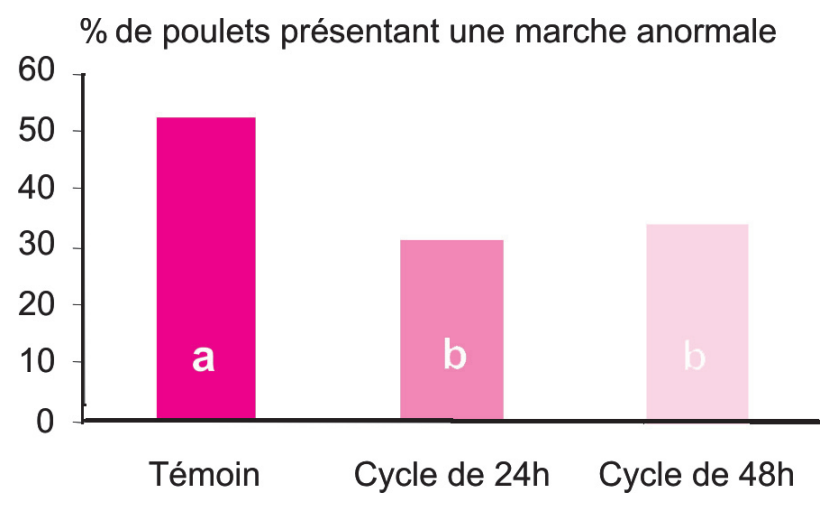

Ce type d'alimentation permet de réduire la croissance avant l'âge de 3 semaines, d'augmenter l'activité générale des animaux et de diminuer le nombre d'animaux présentant des anomalies de la marche par rapport aux témoins (Leterrier et al 2006).

l'Aviculture (ITAVI) en collaboration avec des industriels français.

L'éclairage influence la croissance, l'activité des poulets et les troubles locomoteurs (Gordon 1994, Buyse et al 1996). Comparée à une période de $24 \mathrm{~h}$ de lumière par jour, une période plus réduite ralentit la croissance des poulets et diminue les problèmes locomoteurs et métaboliques, sans que cela ne compromette les performances. Supérieure à $22 \mathrm{~h}$ par jour, elle provoque des troubles du développement du système visuel. L'intensité lumineuse est également à considérer. Très faible, elle entraîne un mauvais développement du système visuel ; faible, elle diminue l'activité des poulets et favorise de ce fait l'apparition de problèmes au niveau des pattes ; forte, elle stimule l'activité des poulets, mais les conséquences sur leurs performances (dont la croissance) varient selon les auteurs (Buyse et al 1996).

La croissance élevée des poulets, fortement liée à la sélection génétique, est un important facteur de risque pour les problèmes locomoteurs et métaboliques. Une diminution de la vitesse de croissance, par la conduite d'élevage ou la voie génétique, diminuerait la fréquence d'apparition de ces problèmes de santé ou les risques de les augmenter. Par ailleurs, la voie génétique ouvre des perspectives pour améliorer le bien-être des poulets puisque la qualité des aplombs, un des facteurs responsable des troubles locomoteurs, est héritable et donc modifiable par sélection (Le Bihan-Duval et al 1996), ainsi que l'adaptabilité aux conditions de l'environnement comme, par exemple, la sensibilité au stress (Craig et Muir 1998, Mignon-Grasteau et Faure 2002).

\section{2 / Bien-être des reproduc- teurs de type chair}

Les reproducteurs à l'origine des poulets de chair élevés pour la consommation de viande sont élevés en groupe de quatre à huit mille individus. Ils sont élevés en sexe séparé jusqu'à l'âge de la reproduction, au sol (sur de la litière), en claustration et nourris avec une quantité de nourriture sévèrement rationnée.

\section{1 / Problèmes de bien-être rencontrés}

Les problèmes de bien-être rencontrés chez les reproducteurs de poulets de chair sont directement liés, pour leur majorité, à la vitesse de croissance élevée imposée par les contraintes économiques pour réduire le temps d'élevage. Lorsqu'ils sont nourris à volonté, les reproducteurs à fort potentiel de croissance présentent de très mauvaises performances de reproduction, autant chez le mâle que chez la femelle, et une forte mortalité. Celle-ci résulte de fréquentes anomalies cardiaques qui se traduisent par des morts subites ou l'apparition d'ascite. Apparaissent également des problèmes articulaires susceptibles d'entraîner des phénomènes douloureux. Pour contrer ces effets néfastes de la forte vitesse de croissance, des programmes lumineux sont utilisés, qui limitent la croissance précoce et permettent d'obtenir des performances de reproduction suffisantes. Ils sont complétés par des programmes de rationnement alimentaire drastiques, mis en place pour freiner la croissance avant la maturité sexuelle et maintenir les performances ultérieures. Les animaux rationnés reçoivent ainsi 40 à $50 \%$ de la quantité d'aliments consommée par les animaux nourris ad libitum. Ce rationnement a pour conséquence une modification du comportement des animaux qui surconsomment l'eau, présentent des activités stéréotypées où ils piquent fréquemment les murs ou la litière avec leur bec (Mench 2002). De nombreuses expériences ont montré que les animaux restreints avaient faim, manifestaient des comportements de frustration et présentaient des modifications physiologiques importantes (réduction de la fréquence cardiaque et augmentation du cortisol plasmatique), tandis que l'activité motrice était globalement très augmentée (de Jong et al 2002).

\section{2 / Solutions en cours d'élabo- ration}

Deux voies majeures ont été testées ces dernières années pour améliorer le bien-être des reproducteurs : 1) Modifier les programmes de rationnement soit en utilisant un aliment dilué (mélangé à des composants alimentaires non nutritifs) de manière à limiter les phénomènes de frustration et réduire les comportements stéréotypés, soit en adaptant mieux les phases de restriction à l'âge et au sexe des animaux, 2) Rechercher des génotypes supportant le rationnement avec de faibles perturbations comportementales et métaboliques.

L'étude des dilutions alimentaires a donné des résultats divers. Certains auteurs ont vu une réduction des signes de frustration chez les reproducteurs (Hocking et al 2004) ; d'autres, par contre, considèrent que la frustration n'est pas supprimée puisque la satiété n'est pas atteinte (Savory et al 1996). Des essais montrent que chez les femelles, la restriction alimentaire n'est plus nécessaire après 15 semaines d'âge pour obtenir de bonnes performances de reproduction et que les mâles pourraient être restreints moins sévèrement (Mench 2002). Néanmoins, ces adaptations des programmes alimentaires ne sont utilisables qu'avant la maturité sexuelle. Ils sont difficiles à mettre en place lors de la phase de reproduction car ils imposent d'élever séparément les animaux des deux sexes, donc de recourir à l'insémination artificielle et à l'élevage en cage, ce qui entraînerait de nouveaux problèmes de bien-être et de rentabilité économique.

Des recherches récentes menées à l'INRA de Tours montrent qu'il est possible, avec certaines lignées naines, 
d'obtenir de bonnes performances de reproduction sans observer de modifications comportementales indicatrices de stress chronique (Heck et al 2004, Picard et Bouchot 2004). Dans d'autres lignées, le comportement agressif des mâles vis-à-vis des femelles est plus faible (Mc Gary et al 2003).

\section{3 / Législation future}

La future directive sur la protection des poulets de chair ne concernera pas les reproducteurs. Si le projet actuel est maintenu, il constituera une démarche novatrice reposant non plus sur une obligation de moyen, comme cela était le cas dans les directives précédentes, mais sur une obligation de résultat. Dans cette approche, qui prend pour modèle un plan de surveillance mis en place en Suède, la densité d'élevage serait modulée en fonction des taux de mortalité et de pododermatites mesurés à l'abattoir sur les lots précédents.
Cette évaluation minimaliste du bienêtre qui ne tient compte que d'un type de lésion est discutable (Veissier et al 2007). Par exemple, des résultats préliminaires obtenus en élevage commercial sur 30 lots, dans le cadre d'un projet réalisé conjointement entre l'ITAVI, l'INRA et l'Agence Française de Sécurité Sanitaire des Aliments (AFSSA), montrent qu'il n'y aurait pas systématiquement de lien entre lésions du bréchet et pododermatites (Colas et al 2005). On ne peut donc pas résumer le bien-être à la seule quantification des pododermatites.

\section{Conclusion}

Plusieurs points critiques en terme de bien-être ont été identifiés chez les poulets de chair et les reproducteurs à l'origine de ce croisement terminal. Ils relèvent de troubles comportementaux ou de problèmes de santé. Des solutions peuvent être apportées en utilisant des génotypes plus appropriés et en contrôlant certains facteurs liés aux conduites d'élevage : chargement, éclairage, alimentation, complexité de l'environnement d'élevage. Chez les reproducteurs, des solutions restent à trouver pour éviter l'important rationnement alimentaire et ses effets négatifs sur le bien-être. Il n'en reste pas moins que, dans ces productions, les contraintes économiques limitent la marge de manipulation des facteurs majeurs influençant le bien-être : vitesse de croissance et chargement. La directive européenne en préparation concernant la protection des poulets de chair imposera de nouvelles contraintes qui auront certainement des conséquences économiques. Son efficacité en terme d'amélioration des conditions d'élevage passe par la qualité des indicateurs de bien-être utilisés, sujet sur lequel travaillent plusieurs équipes, en France (projet ITAVI-INRA-AFSSA) comme en Europe (projet Welfare Quality $^{\circledR}$ ).

\section{Références}

Anonyme, 2000. The welfare of chickens kept for meat production (broilers). Report of the scientific committee on animal health and animal welfare (adopted 21 March 2000), European commission, Health and consumer protection directorate-general. 149p. http://europa.eu.int/ comm/food/fs/sc/scah/outcome en.html

Arnould C., Faure J.M., 2003. Répartition spatiale et activité de poulets de chair élevés en grand groupe. In : L'éthologie appliquée aujourd'hui. Vol I : Bien-être, élevages et expérimentation. Baudoin C. (Ed), Editions ED, LevalloisPerret, France, 1, 73-81.

Arnould C., Faure J.M., 2004. Use of pen space and activity of broiler chickens reared at two different densities. Appl. Anim. Behav. Sci., $87,155-170$.

Arnould C., Bizeray D., Faure J.M., Leterrier C., 2004. Effects of the addition of sand and string in pens on the use of space, activity, tarsal angulations and bone composition in broiler chickens. Anim. Welf., 13, 87-94.

Bouvarel I., Barrier-Guillot B., Larrouche P., Boutten B., Leterrier C., Merlet F., Vilarino M., Roffidal L., Tesseraud S., Castaing J., Picard M., 2004. Sequential feeding programs for broiler chickens : twenty-four and forty-eight hour cycles. Poult. Sci., 83, 49-60.

Buyse J., Simons P.C.M., Boshouwers F.M.G, Decuypere E., 1996. Effect of intermittent lighting, light intensity and source on the performance and welfare of broilers. World's Poult. Sci. J., 52, 121-130.

Colas M., Mirabito L., Arnould C., Michel V., 2005. Première approche des relations entre lésions indicatrices de bien-être mesurées à l'abattoir chez le poulet. Session régionale d'information volailles de chair dans le Sud-Est, 30 novembre 2005, Valence, ITAVI, France, 8p.
Commission européenne, 2005. Proposition de Directive du conseil fixant des règles minimales de protection des poulets destinés à la production de viande. COM(2005) 221 final, 28p. http://ec.europa.eu/food/animal/welfare/farm/pr oposal_FR.pdf

Cornetto T., Estevez I., 2001. Influence of vertical panels on use of space by domestic fowl. Appl. Anim. Behav. Sci., 71, 141-153.

Cornetto T., Estevez I., Douglass, L.W., 2002. Using artificial cover to reduce aggression and disturbances in domestic fowl. Appl. Anim. Behav. Sci., 75, 325-336.

Craig J., Muir W., 1998. Genetics and the behavior of chickens: welfare and productivity. In: Genetics and the behaviour of domestic animals. Grandin T. (Ed), Academic press, London, UK, 265-297.

Dawkins M.S., Donnelly C.A., Jones T.A., 2004. Chicken welfare is influenced more by housing conditions than by stocking density. Nature, 427, 342-344.

De Jong I C, van Voorst S, Ehlhardt D A. Blokhuis H.J., 2002. Effects of restricted feeding on physiological stress parameters in growing broiler breeders. Br. Poult. Sci., 43, 157-168

Elwinger K., 1995. Broiler production under varying population densities - a field study. Arch. Geflügelk., 59, 209-215.

Fraysse V., Mirabito L., Arnould C., 2001. Utilisation de l'espace par des poulets de chair «standards» et activité au niveau des abreuvoirs et des mangeoires selon leur localisation. 4 èmes Journ. Rech. Avicole, 27-29 mars 2001 Nantes, France, 105-108.

Gordon S.H., 1994. Effects of daylength and increasing daylength programmes on broiler welfare and performance. World's Poult. Sci. J. 50, 269-282.

Heck A., Onagbesan O., Tona K., Metayer S., Puterflam J., Jego Y., Trevidy J.J., Decuypere E., Williams J., Picard M., Bruggeman V., 2004. Effects of ad libitum feeding on performance of different strains of broiler breeders. Br. Poult. Sci., 45, 685-703.

Hocking P. M., Zaczek V., Jones E.K.M., MacLeod M.G., 2004. Different sources of fibre may improve the welfare of female broiler breeders. Br. Poult. Sci., 45, 9-19.

Kells A., Dawkins M.S., Cortina Borja M. 2001. The effect of a «freedom food» enrichment on the behaviour of broilers on commercial farms. Anim. Welf., 10, 347-356.

Le Bihan-Duval E., Beaumont C., Colleau J.J., 1996. Genetic parameters of the twisted legs syndrome in broiler chickens. Genet. Sel. Evol., 28, 177-195.

Leterrier C., Perrot P., Favreau F., Constantin P., Bouvarel I., Lessire M., Picard M., 2006. Sequential feeding with low- and high-lysine diets increases activity and improves gait score in broiler chickens. XII Eur. Poult. Conf., Verona, Italy, 10-14 septembre 2006, 581 .

Martrenchar, A., Morisse, J.P., Huonnic, D. Cotte, J.P., 1997. Influence of stocking density on some behavioural, physiological and productivity traits of broilers. Vet. Res., 28, 473480 .

Mc Gary S., Estevez I., Russek-Cohen E., 2003. Reproductive and agressive behavior in male broiler breeder with varying fertility level. Appl. Anim. Behav. Sci., 82, 29-44.

McLean J.A., Savory C.J., Sparks N.H.C., 2002. Welfare of male and female broiler chickens in relation to stocking density, as indicated 
by performance, health and behaviour. Anim. Welf., 11, 55-73.

Mench J.A., 2002. Broiler breeders, feed restriction and welfare. World's Poult. Sci. J., 58, 23-29.

Mignon-Grasteau S., Faure J.M., 2002. Génétique et adaptation : le point des connaissances chez les volailles. INRA Prod. Anim., 15, 357-364.
Picard M., Bouchot C., 2004. Broiler Breeder Paradox. Results of the European project QLK5CT-2001-01732. CDROM SRA. INRA, Tours, France.

Savory C.J., Hocking P.M., Mann J.S., Maxwell M.H., 1996. Is broiler breeder welfare improved by using qualitative rather than quantitative food restriction to limit growth rate? Anim. Welf., 5, 105-127.
Terlouw E.M.C., Arnould C., Auperin B. Berri C., Le Bihan-Duval E., Lefevre F., Lensink J., Mounier L., 2007. Impact des conditions de pré-abattage sur le stress et le bien-être des animaux d'élevage. INRA Prod. Anim., 20, 92-100.

Veissier I., Beaumont C., Lévy F., 2007. Les recherches sur le bien-être animal : buts, méthodologies et finalité. INRA Prod. Anim., 20, 3-10.

\title{
Résumé
}

L'élevage des poulets de chair s'est intensifié ces dernières décennies et de nombreux problèmes de bien-être animal sont apparus, en particulier chez les poulets standards et les reproducteurs à l'origine de ces croisements. Les poulets standards ont un niveau d'activité très faible et ils sont notamment atteints de troubles locomoteurs, de lésions cutanées et de pathologies cardio-vasculaires. Des solutions à ces altérations du bien-être peuvent être apportées en contrôlant des facteurs liés aux conduites d'élevage (densité, ventilation, aménagement de l'environnement, alimentation, lumière) et en utilisant des génotypes plus appropriés. Les reproducteurs, quant à eux, sont soumis à des programmes de rationnement sévères, entraînant de la faim et une frustration que l'on essaye de limiter par des moyens génétiques et alimentaires. Une directive européenne sur la protection des poulets de chair devrait voir le jour prochainement. Elle devrait reposer sur une obligation de résultats : la densité d'élevage pourrait être modulée en fonction des taux de mortalité et de lésions cutanées (pododermatites) mesurés à l'abattoir sur les bandes précédentes.

\begin{abstract}
Welfare of chickens reared for meat production

Chicken farming has become more intensive over the last decades and numerous welfare problems have arisen, especially in broilers and broiler breeders. The activity level of broiler chickens is very low and they are often affected by skin lesions, leg problems or cardiovascular disease. Improvement in welfare can be achieved by better control of certain rearing factors (stocking density, ventilation, complexity of the environment, feeding, light) and by using genotypes that are better suited to the rearing environment. Broiler breeders are subject to severe feeding restrictions inducing hunger and frustration that can be alleviated by genetic and nutritional means. A directive laying down minimum rules for the protection of chickens kept for meat production will be published shortly. The maximum stocking density allowed could be adjusted according to rates of mortality and skin lesions (pododermatitis) measured at the slaughterhouse.
\end{abstract}

ARNOULD C., LETERRIER C., 2007. Bien-être animal en élevage de poulets de chair. INRA Prod. Anim., 20, 41-46. 
